\title{
Sex estimation using cervical dental measurements in an archaeological population from Iran
}

\author{
Seyedeh M. $\operatorname{Kazzazi}^{1}$ • Elena F. Kranioti ${ }^{1}$
}

Received: 1 February 2016 / Accepted: 22 July 2016 / Published online: 6 August 2016

(C) The Author(s) 2016. This article is published with open access at Springerlink.com

\begin{abstract}
Sex estimation of skeletal remains is one of the major components of forensic identification of unknown individuals. Teeth are a potential source of information on sex and are often recovered in archaeological or forensic contexts due to their post-mortem longevity. Currently, there is limited data on dental sexual dimorphism of archaeological populations from Iran. This paper represents the first study to provide a dental sex estimation method for Iron Age populations. The current study was conducted on the skeletal remains of 143 adults from two Iron Age populations in close temporal and geographic proximity in the Solduz Valley (West Azerbaijan Province of Iran). Mesiodistal and buccolingual cervical measurements of 1334 maxillary and mandibular teeth were used to investigate the degree of sexual dimorphism in permanent dentition and to assess their applicability in sex estimation. Data was analysed using discriminant function analysis (SPSS 23), and posterior probabilities were calculated for all produced formulae. The results showed that incisors and canines were the most sexually dimorphic teeth, providing percentages of correct sex classification between 86.4 and $100 \%$ depending on the measurement used. The combination of canines and other teeth improved significantly the level of correct sex classification. The highest percentages of sex classification were obtained by the combination of canines and incisors $(100 \%)$ and canines and molars $(92.3 \%)$. The present study provided the first reference standards for sex estimation
\end{abstract}

Seyedeh M. Kazzazi

S.M.Kazzazi@sms.ed.ac.uk

1 Edinburgh Unit for Forensic Anthropology, School of History Classics and Archaeology, University of Edinburgh, William Robertson Wing, Old Medical School, 4 Teviot place, Edinburgh EH8 9AG, UK using odontometric data in an Iranian archaeological population. Cervical measurements were found to be of value for sex assessment, and the method presented here can be a useful tool for establishing accurate demographic data from skeletal remains of the Iron Age from Iran.

Keywords Cervical mesiodistal · Cervical buccolingual · Sexual dimorphism

\section{Introduction}

In human populations, males and females normally differ in dental size. On average, males have larger teeth than females and this characteristic could be used in sex estimation (Garn et al. 1964, 1966; Ditch and Rose 1972; Kieser et al. 1985; Hattab et al. 1997; Işcan and Kedici 2003; Hassett 2011; Viciano et al. 2015). The most commonly reported tooth measurements for sex estimation are the maximum mesiodistal and buccolingual crown measurements (Black 1978; Hattab et al. 1997; Kondo and Townsend 2004; Acharya and Mainali 2007; Pereira et al. 2010; Mitsea et al. 2014; Gonçalves et al. 2014; Sharma et al. 2015). These measurements, however, are difficult to obtain in worn teeth or crowns that are embedded in the jaw. To solve this issue, alternative measurements of cervical tooth diameters were proposed by Hillson et al. (2005). Cervical measurements are useful in the studies of prehistorical skeletal remains as they allow for the inclusion of teeth with alterations on the crown due to wear, pathology (e.g. caries), cultural modification or post-mortem damage. This allows a larger dataset to be obtained with a broader range of ages represented.

Studies on sexual dimorphism in cervical mesiodistal and buccolingual measurements have demonstrated that the canine is the most sexual dimorphic tooth (Starp 1990; Ellendt 1993; Alt et al. 1998, Vodanovic et al. 2007, Viciano et al. 
2011, 2013, 2015; Hassett 2011, Mujib et al. 2014, Viciano et al. 2015). This is similar to the conclusion reached in the previous studies on crown mesiodistal and buccolingual measurements (Garn et al. 1966; Yuen et al. 1997; Ișcan and Kedici 2003; Vodanovic et al. 2007; Cardoso 2008; Zorba et al. 2011; Khamis et al. 2014). In addition, studies on cervical measurements of molars suggest that the first and second molars are also among the most dimorphic teeth (Alt et al. 1998; Starp 1990; Ellendt 1993; Zorba et al. 2012, 2013).

A large number of studies have demonstrated that the degree of sexual dimorphism in teeth varies between populations (Bishara et al. 1989; Ateş et al. 2006; Prabhu and Acharya 2009) as a result of genetic and environmental factors (Kieser 1990; Hughes and Townsend 2013). Therefore, the collection of data from different populations is important for dental sexual dimorphism. As currently, there is no reference odontometric data from Iranian archaeological populations, the present study contributes to the development of standards for sex estimation.

The Hasanlu population, which is the focus of this study, represents a distinctive sample of anthropological and archaeological value due to the unusual way in which the sample was formed. Most of the biological remains belong to victims of an instantaneous catastrophe and so the paleodemographic data is synchronous. Moreover, the Hasanlu osteological collection represents one of only a few well-preserved skeletal collections from Iron Age Iran.

The present study aims to examine the level of sexual dimorphism in permanent teeth of Iranian archaeological populations using cervical mesiodistal and buccolingual diameters and to assess the applicability of cervical measurements in sex estimation based on discriminant function analysis.

\section{Material and methods}

\section{Material}

This study is conducted on the skeletal remains of 107 individuals from Hasanlu site (West Azerbaijan, Iran) dating from 1450 to $800 \mathrm{BC}$ and 36 individuals from Dinkha Tepe (15 mi west of the Hasanlu site) dating from 1350 to $800 \mathrm{BC}$. Hasanlu is the largest and most important archaeological site in Gadar River Valley in north western Iran. This settlement was destroyed by fire during a battle probably with Urartians around 800 BC (Dyson 1965).

Hasanlu and Dinkha Tepe are located $15 \mathrm{mi}$ apart, and they are determined to be of similar age (Muscarella 1988). Dinkha II and III burials (1350-800 BC) contained cultural material paralleling that of Hasanlu period IV and V (1450-800 BC) (Muscarella 1974, 1988), which are the main focuses of this study.
The skeletons of the Hasanlu and Dinkha Tepe individuals are stored in the University of Pennsylvania's Museum of Archaeology and Anthropology (UPM), USA. In total, 1334 maxillary and mandibular teeth of 143 skeletons ( 95 males, 48 females) are studied. The sex of the skeletons was estimated using the morphological features of the pelvis (differences in the ventral arch, subpubic concavity, and ischiopubic ramus (Phenice 1969)) and skull (nuchal crest, mastoid process, glabella, supraorbital margin, mental eminence, and shape of orbit (Walker 2008)). All the 143 individuals used in this study are adults aged between 20 and 65 years for both males and females. The age at death of the individuals was estimated based on the degree of dental wear (Miles 2001; Buikstra and Ubelaker 1994), changes in the pubic symphyseal face (Brooks and Suchey 1990) and ilium auricular area (Buckberry and Chamberlain 2002), and the closure of cranial sutures (Meindl and Lovejoy 1985).

\section{Data acquisition}

Tooth measurements are taken on the loose teeth as well as on teeth intact in the jaw using the Hillson-Fitzgerald Paleotech dental calliper (modified digital Mitutyo needlepoint calliper) that was specifically developed to measure the diameters of teeth even if they are in the jaw. Mesiodistal cervical measurements are taken as the greatest mesiodistal dimension parallel to the occlusal and buccal surface measured in the cervical part of the tooth crown (Vodanovic et al. 2007), and buccolingual cervical measurements are taken as the maximum measurement at the cement-enamel junction from buccal to lingual surface (Hillson et al. 2005).

Dental measurements are taken from the left and right maxillary and mandibular teeth. To avoid the possibility of incorrect measurements, the samples with caries, heavy calculus deposits, and hypoplastic defects along cemento-enamel junction were excluded. Due to the small number of maxillary third molars, as well as their high degree of variation in size (Townsend et al. 2016), measurements for this tooth were excluded from the discriminant function analysis. In total, 1324 teeth -1007 teeth from Hasanlu and 317 teeth from Dinkha Tepe - are measured (Table 2).

\section{Statistical analysis}

Bilateral asymmetry of right and left side in the entire sample is tested using a paired $t$ test.

To assess the intra-observer error, mesiodistal and buccolingual cervical measurements are collected from 619 randomly selected teeth from the original sample at a different time by a single observer (SMK). Technical error of measurements (TEM), relative technical error of measurements (rTEM), and the coefficient of reliability (Ulijaszek and Kerr 
1999) are used to determine the differences between two sets of measurements.

A one-way ANOVA is used to compare the mean differences between males and females. An independent sample $t$ test is carried out to determine if there was any statistical significant differences between Hasanlu and Dinkha Tepe collections. The percentage of sexual dimorphism for each measurement is also calculated using the Garn et al. (1967) formula: (male mean-female mean) $\times 100 / 2$.

Univariate discriminant function analysis is used for each variable. Stepwise discriminant function analysis is used to determine which variables best discriminate males and females. Separate discriminant analysis is conducted for cervical measurements of teeth, separately by tooth class (incisor, canine, premolar, and molar) and position (maxillary and mandibular). Many studies have shown that canines are the most sexually dimorphic teeth; therefore, we added maxillary and mandibular canines to each function to indicate whether the classification success would increase. In addition, in order to increase the applicability of technique where the dentitions are not well preserved, the analysis is also conducted for each tooth separately.

Bootstrapping is used in all cases to account for possible biases due to the small sample size.

A leave-one-out classification procedure is also used to demonstrate the accuracy rate of the original sample and the one created by cross-validation. Posterior probabilities of each individual are also calculated as they reflect the affinity of each case to be reassigned to the original group according to the value of the discriminant score (Kranioti et al. 2008). Statistical analysis is conducted using SPSS 23 software programme.

\section{Results}

Mean differences of all measurements between left and right maxillary and mandibular teeth were found to be statistically insignificant $(p>0.05)$. As a principle, we used measurements from the right maxillary and mandibular teeth for the analysis. In the case of a missing value from the right side, the left antimere was substituted.

Table 1 shows the differences between the mean values, TEM, rTEM, and the coefficient of reliability $(R)$ for mesiodistal and buccolingual measurements of anterior and posterior teeth. The values of the TEM and rTEM were low, varying between $0.02-0.07 \mathrm{~mm}$ and $0.26-0.72 \mathrm{~mm}$, respectively, with the exception of the mesiodistal measurement of mandibular second incisor $(1.81 \mathrm{~mm})$. $R$ values ranged between 0.99 and 1.00 . The only variable that showed a coefficient of reliability less than $99 \%$ was the mesiodistal measurement of mandibular incisor (0.96).
In the mesiodistal and buccolingual measurements, both maxillary and mandibular teeth showed no statistically significant differences between the mean values of Hasanlu and Dinkha Tepe skeletons $(p<0.05)$ (Table 2$)$, except for mesiodistal maxillary M3. Accordingly, the two samples were subsequently pooled to increase sample size for analysis.

The results of the one-way ANOVA indicated that the differences between male and female mean values were significant for all measurements $(p<0.001)$ except for mesiodistal upper M3. Table 3 shows descriptive statistics, associated univariate $F$-ratio, and percentage of sexual dimorphism. The most sexually dimorphic measurements was the mesiodistal diameter of mandibular canine with 14.99 percentage of dimorphism followed by the mesiodistal diameter of maxillary canine and maxillary second molar each with 13.93 and 13.40 percentage of dimorphism respectively (Table 3 ).

Discriminant function analysis was carried out for samples $>20$ individuals that had relatively equal size groups. Maxillary M3 was removed from the discriminant analysis due to the small number of data, and mandibular M3 was also excluded from functions 5 and 9 to increase the sample size. Tables 4, 5, and 6 show the tooth variables in each function that were used in discriminant function analysis. Functions 1 to 9 (Table 4) demonstrate the results of stepwise discriminant function analysis using cervical measurements of each tooth type. Maxillary and mandibular canines also added to functions 6 to 9 to examine whether the classification accuracy would increase. Functions 10 to 21 also demonstrate the results of discriminant function analysis for each tooth separately (Tables 5 and 6). $F$ value gives an indication of the contribution of variables entered in the equation to separate sexes. Unstandardised (raw) coefficient is used to calculate the discriminant scores. The sex of the individual can be assessed by multiplying the tooth measurement with its respective unstandardised coefficient and added to the constant. The sectioning point was set to zero for all the functions; therefore, if the value obtained is greater than the sectioning point of zero, the individual is considered male, and if less than zero, the individual is considered female.

Table 7 shows the classification accuracy of all functions. The best classification accuracy $(100 \%)$ was achieved with functions 1 and 6, which used the anterior teeth. The combination of maxillary and mandibular M1 and M2 with maxillary and mandibular canines (function 9) gave the next best classification $(92.3 \%)$, followed by maxillary and mandibular canines (function 2: $86.4 \%$ ). The canines, which showed the highest percentage of sexual dimorphism (Table 3), were added to functions 6 to 9 . The classification accuracy significantly improved in all functions particularly in function 8 (Table 7). Mandibular M3 was removed from the analysis, which increased the sample size to 52 and 39 and also the classification accuracy to 82.7 and $92.3 \%$ for functions 5 and 9 , respectively. The best discriminant function for the 
Table 1 TEM and rTEM results evaluating intra-observer error in cervical tooth measurements

\begin{tabular}{|c|c|c|c|c|c|c|c|c|c|}
\hline \multirow[t]{2}{*}{ Measurements } & \multirow[t]{2}{*}{$\mathrm{N}$} & \multicolumn{2}{|c|}{ Measurement 1} & \multicolumn{2}{|c|}{ Measurement 2} & \multirow[t]{2}{*}{ Diff } & \multirow[t]{2}{*}{ TEM } & \multirow[t]{2}{*}{ rTEM } & \multirow[t]{2}{*}{$R$} \\
\hline & & Mean & SD & Mean & SD & & & & \\
\hline \multicolumn{10}{|l|}{ Mesiodistal } \\
\hline UI1 & 36 & 6.18 & 0.59 & 6.19 & 0.59 & -0.01 & 0.03 & 0.45 & 1.00 \\
\hline LI1 & 25 & 3.51 & 0.18 & 3.51 & 0.18 & 0.00 & 0.02 & 0.58 & 0.99 \\
\hline UI2 & 36 & 4.77 & 0.44 & 4.77 & 0.44 & 0.00 & 0.03 & 0.61 & 1.00 \\
\hline LI2 & 39 & 3.86 & 0.36 & 3.88 & 0.36 & -0.02 & 0.07 & 1.81 & 0.96 \\
\hline $\mathrm{UC}$ & 37 & 5.48 & 0.57 & 5.48 & 0.57 & 0.00 & 0.03 & 0.53 & 1.00 \\
\hline $\mathrm{LC}$ & 48 & 5.30 & 0.60 & 5.31 & 0.60 & -0.01 & 0.03 & 0.49 & 1.00 \\
\hline UP3 & 38 & 4.59 & 0.38 & 4.60 & 0.40 & -0.01 & 0.03 & 0.72 & 0.99 \\
\hline LP3 & 50 & 4.82 & 0.33 & 4.83 & 0.33 & -0.01 & 0.03 & 0.55 & 0.99 \\
\hline UP4 & 42 & 4.84 & 0.82 & 4.85 & 0.82 & -0.01 & 0.03 & 0.65 & 1.00 \\
\hline LP4 & 38 & 5.08 & 0.87 & 5.08 & 0.86 & 0.00 & 0.02 & 0.47 & 1.00 \\
\hline UM1 & 48 & 7.66 & 0.56 & 7.66 & 0.55 & 0.00 & 0.03 & 0.39 & 1.00 \\
\hline LM1 & 31 & 8.85 & 0.52 & 8.86 & 0.53 & -0.01 & 0.02 & 0.26 & 1.00 \\
\hline UM2 & 42 & 7.55 & 0.70 & 7.56 & 0.71 & -0.01 & 0.03 & 0.45 & 1.00 \\
\hline LM2 & 22 & 9.01 & 0.65 & 9.02 & 0.64 & -0.02 & 0.03 & 0.36 & 1.00 \\
\hline $\mathrm{UM}^{\mathrm{a}}$ & 18 & 6.88 & 1.00 & 6.88 & 1.02 & 0.00 & 0.04 & 0.54 & 1.00 \\
\hline LM3 & 31 & 8.80 & 0.85 & 8.81 & 0.86 & 0.00 & 0.03 & 0.36 & 1.00 \\
\hline \multicolumn{10}{|l|}{ Buccolingual } \\
\hline UI1 & 36 & 6.32 & 0.62 & 6.33 & 0.62 & -0.01 & 0.03 & 0.48 & 1.00 \\
\hline LI1 & 22 & 5.64 & 0.37 & 5.64 & 0.37 & 0.00 & 0.03 & 0.49 & 0.99 \\
\hline UI2 & 36 & 5.64 & 0.43 & 5.64 & 0.43 & 0.00 & 0.02 & 0.44 & 1.00 \\
\hline LI2 & 39 & 6.04 & 0.34 & 6.05 & 0.35 & -0.01 & 0.03 & 0.47 & 0.99 \\
\hline UC & 37 & 7.81 & 0.80 & 7.81 & 0.80 & 0.00 & 0.03 & 0.33 & 1.00 \\
\hline $\mathrm{LC}$ & 48 & 7.41 & 0.56 & 7.41 & 0.56 & 0.00 & 0.02 & 0.31 & 1.00 \\
\hline UP3 & 38 & 8.07 & 0.61 & 8.08 & 0.61 & -0.01 & 0.03 & 0.38 & 1.00 \\
\hline LP3 & 50 & 6.72 & 0.41 & 6.72 & 0.42 & 0.00 & 0.03 & 0.45 & 0.99 \\
\hline UP4 & 42 & 8.29 & 0.83 & 8.28 & 0.82 & 0.01 & 0.03 & 0.39 & 1.00 \\
\hline LP4 & 38 & 7.14 & 0.57 & 7.14 & 0.58 & 0.00 & 0.03 & 0.35 & 1.00 \\
\hline UM1 & 41 & 9.93 & 0.56 & 9.93 & 0.56 & -0.01 & 0.03 & 0.29 & 1.00 \\
\hline LM1 & 40 & 8.71 & 0.63 & 8.71 & 0.61 & 0.00 & 0.03 & 0.33 & 1.00 \\
\hline UM2 & 39 & 9.80 & 1.03 & 9.81 & 1.03 & -0.01 & 0.03 & 0.32 & 1.00 \\
\hline LM2 & 37 & 8.18 & 0.77 & 8.17 & 0.76 & 0.01 & 0.03 & 0.38 & 1.00 \\
\hline $\mathrm{UM}^{\mathrm{a}}$ & 25 & 9.60 & 0.82 & 9.59 & 0.81 & 0.01 & 0.03 & 0.36 & 1.00 \\
\hline LM3 & 31 & 8.10 & 0.70 & 8.11 & 0.70 & -0.01 & 0.03 & 0.39 & 1.00 \\
\hline
\end{tabular}

$U$ upper, $L$ lower, $I$ incisor, $C$ canine, $P$ premolar, $M$ molar

${ }^{\mathrm{a}} \mathrm{M}^{3}$ was excluded from the discriminant function analysis single tooth measurements used mandibular I 2 to differentiate between males and females and reached $87.9 \%$ accuracy followed by maxillary and mandibular canines ( 86.9 and 86.3 , respectively). In all functions, the accuracy in males was significantly greater than females (Table 7). Crossvalidation accuracy was close to the original classification accuracy in all cases (Table 7).

Figures 1 and 2 demonstrate the probability levels of correct group assessment according to the discriminant scores of each individual. For example, if a discriminant score based on the stepwise analysis of cervical measurements of mandibular canine (function 15 ) is 3.26 (x coordinate), the posterior probability of that individual coming from a male group is $99.9 \%$ (y coordinate).

\section{Discussion}

Dental sexual dimorphism has been long acknowledged (Garn et al. 1964, 1966; Ditch and Rose 1972; Bishara et al. 1989; 
Table 2 Independent Student's $t$ test comparing the means between Hasanlu and Dinkha Tepe collections

\begin{tabular}{|c|c|c|c|c|c|c|c|c|}
\hline \multirow[t]{2}{*}{ Measurements } & \multicolumn{3}{|c|}{ Hasanlu } & \multicolumn{3}{|c|}{ Dinkha Tepe } & \multirow{2}{*}{$\begin{array}{l}t \\
\text { value* }\end{array}$} & \multirow{2}{*}{$\begin{array}{l}p \\
\text { value }\end{array}$} \\
\hline & $n$ & Mean & SD & $n$ & Mean & SD & & \\
\hline \multicolumn{9}{|l|}{ Mesiodistal } \\
\hline UI1 & 44 & 6.30 & 0.55 & 8 & 6.05 & 0.42 & 1.21 & 0.23 \\
\hline LI1 & 55 & 3.52 & 0.26 & 20 & 3.49 & 0.21 & 0.39 & 0.70 \\
\hline UI2 & 43 & 4.85 & 0.44 & 12 & 4.62 & 0.47 & 1.64 & 0.10 \\
\hline LI2 & 68 & 3.85 & 0.32 & 23 & 3.97 & 0.33 & -1.48 & 0.14 \\
\hline UC & 64 & 5.60 & 0.50 & 20 & 5.53 & 0.54 & 0.55 & 0.58 \\
\hline $\mathrm{LC}$ & 75 & 5.31 & 0.50 & 27 & 5.43 & 0.60 & -0.99 & 0.32 \\
\hline UP3 & 71 & 4.56 & 0.39 & 16 & 4.58 & 0.50 & -0.20 & 0.84 \\
\hline LP3 & 84 & 4.73 & 0.37 & 27 & 4.84 & 0.37 & -1.41 & 0.16 \\
\hline UP4 & 64 & 4.69 & 0.39 & 19 & 4.76 & 0.44 & -0.71 & 0.48 \\
\hline LP4 & 80 & 4.97 & 0.41 & 28 & 5.13 & 0.39 & -1.75 & 0.08 \\
\hline UM1 & 67 & 7.72 & 0.45 & 17 & 7.73 & 0.36 & -0.14 & 0.88 \\
\hline LM1 & 74 & 8.86 & 0.59 & 22 & 9.00 & 0.53 & -0.89 & 0.38 \\
\hline UM2 & 62 & 7.47 & 0.71 & 20 & 7.71 & 0.66 & -1.30 & 0.20 \\
\hline LM2 & 79 & 8.88 & 0.73 & 28 & 8.95 & 0.62 & -0.45 & 0.65 \\
\hline $\mathrm{UM}^{\mathrm{a}}$ & 33 & 6.80 & 0.60 & 13 & 7.37 & 0.57 & $2.93 * *$ & 0.00 \\
\hline LM3 & 48 & 8.59 & 0.91 & 21 & 8.94 & 0.93 & -1.47 & 0.15 \\
\hline \multicolumn{9}{|l|}{ Buccolingual } \\
\hline UI1 & 44 & 6.29 & 0.40 & 8.00 & 6.07 & 0.56 & 1.32 & 0.19 \\
\hline LI1 & 55 & 5.54 & 0.38 & 20 & 5.45 & 0.34 & 0.90 & 0.37 \\
\hline UI2 & 43 & 5.73 & 0.45 & 12 & 5.52 & 0.46 & 1.41 & 0.16 \\
\hline LI2 & 68 & 6.00 & 0.35 & 23 & 5.97 & 0.39 & 0.31 & 0.76 \\
\hline $\mathrm{UC}$ & 64 & 7.85 & 0.65 & 20 & 7.63 & 0.61 & 1.30 & 0.20 \\
\hline LC & 75 & 7.42 & 0.64 & 27 & 7.45 & 0.71 & -0.22 & 0.82 \\
\hline UP3 & 71 & 7.98 & 0.58 & 16 & 7.75 & 0.56 & 1.47 & 0.15 \\
\hline LP3 & 84 & 6.65 & 0.52 & 27 & 6.82 & 0.66 & -1.36 & 0.18 \\
\hline UP4 & 64 & 8.19 & 0.72 & 19 & 7.99 & 0.51 & 1.14 & 0.26 \\
\hline LP4 & 80 & 7.09 & 0.63 & 24 & 7.35 & 0.57 & -1.82 & 0.07 \\
\hline UM1 & 67 & 9.98 & 0.65 & 17 & 9.87 & 0.47 & 0.68 & 0.49 \\
\hline LM1 & 74 & 8.71 & 0.62 & 22 & 8.78 & 0.65 & -0.45 & 0.65 \\
\hline UM2 & 62 & 9.82 & 0.82 & 20 & 10.05 & 0.70 & -1.11 & 0.27 \\
\hline LM2 & 79 & 8.30 & 0.71 & 28 & 8.40 & 0.69 & -0.65 & 0.49 \\
\hline UM3 & 33 & 9.38 & 0.99 & 13 & 9.22 & 0.95 & -0.49 & 0.63 \\
\hline LM3 & 48 & 7.98 & 0.58 & 21 & 8.03 & 0.59 & -0.33 & 0.74 \\
\hline
\end{tabular}

$U$ upper, $L$ lower, $I$ incisor, $C$ canine, $P$ premolar, $M$ molar

*None of the $t$ values were significant at $p<0.05$

**Statistically significant at $p<0.01$

${ }^{\mathrm{a}} \mathrm{M}^{3}$ was excluded from the discriminant function analysis

Hattab et al. 1997; Hillson et al. 2005; Cardoso 2008; Hassett 2011; Zorba et al. 2013; Viciano et al. 2015; Tuttösí and Cardoso 2015), and studies have demonstrated that dental dimensions can be used to accurately assess sex in living individuals and among skeletal remains (Fig. 3).

Considering that odontometric methods for sex estimation are population-specific, different scholars have undertaken
Table 3 Descriptive statistics, \%SD (sexual dimorphism), and $F$ ratio of the differences between the sexes

\begin{tabular}{|c|c|c|c|c|c|c|c|c|}
\hline \multirow[t]{2}{*}{ Measurements } & \multicolumn{3}{|c|}{ Females } & \multicolumn{3}{|c|}{ Males } & \multirow[t]{2}{*}{$\mathrm{SD} \%$} & \multirow[t]{2}{*}{$F$ ratio } \\
\hline & $n$ & Mean & $\mathrm{SD}$ & $n$ & Mean & SD & & \\
\hline \multicolumn{9}{|l|}{ Mesiodistal } \\
\hline UI1 & 22 & 5.99 & 0.49 & 30 & 6.47 & 0.48 & 8.01 & 12.25 \\
\hline LI1 & 27 & 3.31 & 0.19 & 48 & 3.62 & 0.20 & 9.37 & 41.93 \\
\hline UI2 & 21 & 4.53 & 0.50 & 34 & 4.97 & 0.33 & 9.70 & 14.91 \\
\hline LI2 & 32 & 3.63 & 0.21 & 59 & 4.02 & 0.29 & 10.74 & 44.55 \\
\hline UC & 34 & 5.16 & 0.36 & 50 & 5.88 & 0.36 & 13.93 & 78.52 \\
\hline $\mathrm{LC}$ & 36 & 4.87 & 0.38 & 66 & 5.60 & 0.41 & 14.99 & 76.08 \\
\hline UP3 & 35 & 4.27 & 0.35 & 52 & 4.76 & 0.32 & 11.48 & 44.87 \\
\hline LP3 & 42 & 4.46 & 0.29 & 69 & 4.93 & 0.30 & 10.56 & 67.11 \\
\hline UP4 & 34 & 4.43 & 0.29 & 49 & 4.89 & 0.36 & 10.38 & 39.38 \\
\hline LP4 & 36 & 4.70 & 0.35 & 68 & 5.17 & 0.34 & 10.16 & 43.24 \\
\hline UM1 & 33 & 7.43 & 0.33 & 51 & 7.91 & 0.38 & 6.42 & 35.14 \\
\hline LM1 & 34 & 8.50 & 0.50 & 62 & 9.10 & 0.50 & 7.06 & 31.62 \\
\hline UM2 & 30 & 6.94 & 0.56 & 52 & 7.87 & 0.54 & 13.40 & 54.76 \\
\hline LM2 & 38 & 8.35 & 0.53 & 69 & 9.20 & 0.59 & 10.18 & 54.47 \\
\hline $\mathrm{UM}^{\mathrm{a}}$ & 16 & 6.87 & 0.76 & 30 & 7.00 & 0.58 & 1.89 & $0.41^{\mathrm{b}}$ \\
\hline LM3 & 24 & 8.25 & 1.00 & 45 & 8.94 & 0.79 & 8.36 & $10.05^{\mathrm{c}}$ \\
\hline \multicolumn{9}{|l|}{ Buccolingual } \\
\hline UI1 & 22 & 5.96 & 0.27 & 30 & 6.47 & 0.40 & 8.56 & 27.14 \\
\hline LI1 & 27 & 5.27 & 0.27 & 48 & 5.66 & 0.34 & 7.37 & 26.17 \\
\hline UI2 & 21 & 5.36 & 0.43 & 34 & 5.88 & 0.34 & 9.60 & 23.66 \\
\hline LI2 & 32 & 5.76 & 0.34 & 59 & 6.12 & 0.31 & 6.25 & 25.43 \\
\hline $\mathrm{UC}$ & 34 & 7.28 & 0.51 & 50 & 8.14 & 0.48 & 11.81 & 61.91 \\
\hline $\mathrm{LC}$ & 36 & 6.88 & 0.60 & 66 & 7.73 & 0.46 & 12.35 & 61.62 \\
\hline UP3 & 35 & 7.68 & 0.55 & 52 & 8.12 & 0.54 & 5.73 & 13.45 \\
\hline LP3 & 42 & 6.31 & 0.42 & 69 & 6.92 & 0.50 & 9.67 & 42.87 \\
\hline UP4 & 34 & 7.76 & 0.60 & 49 & 8.42 & 0.59 & 8.51 & 24.81 \\
\hline LP4 & 36 & 6.76 & 0.60 & 68 & 7.35 & 0.61 & 8.67 & 22.80 \\
\hline UM1 & 33 & 9.53 & 0.43 & 51 & 10.24 & 0.57 & 7.42 & 37.80 \\
\hline LM1 & 34 & 8.27 & 0.46 & 62 & 8.97 & 0.56 & 8.46 & 39.11 \\
\hline UM2 & 30 & 9.19 & 0.57 & 52 & 10.28 & 0.61 & 11.86 & 62.11 \\
\hline LM2 & 38 & 7.73 & 0.56 & 69 & 8.65 & 0.58 & 11.90 & 62.15 \\
\hline UM3 & 16 & 8.94 & 0.59 & 30 & 9.54 & 1.07 & 6.79 & $4.39^{\mathrm{d}}$ \\
\hline LM3 & 24 & 7.59 & 0.50 & 45 & 8.21 & 0.50 & 8.17 & 23.73 \\
\hline
\end{tabular}

$U$ upper, $L$ lower, $I$ incisor, $C$ canine, $P$ premolar, $M$ molar.

${ }^{\mathrm{a}} \mathrm{M} 3$ was excluded from the discriminant function analysis

${ }^{\mathrm{b}}$ Not significant

${ }^{\mathrm{c}}$ Significant at $p<0.01$

${ }^{\mathrm{d}}$ Significant at $p<0.05$, all others significant at $p<0.001$

studies on tooth measurements to determine specific standards of group assessment for various populations (Bishara et al. 1989; Alt et al. 1998; Ișcan and Kedici 2003; Ateș et al. 2006; Acharya and Mainali 2007; Hassett 2011; Khamis et al. 2014). The present study is the first reference study for sex estimation using odontometric data in Iranian 
Table 4 Stepwise discriminant function analysis of cervical mesiodistal and buccolingual measurements of all teeth

\begin{tabular}{|c|c|c|c|}
\hline Variables entered ${ }^{\mathrm{a}}$ & Exact $F$ & df & $\begin{array}{l}\text { Raw } \\
\text { coefficien }\end{array}$ \\
\hline \multicolumn{4}{|l|}{ F1: incisors } \\
\hline MDLI2 & 22.05 & 1,25 & 5.25 \\
\hline BLLI2 & 36.74 & 2,24 & 2.97 \\
\hline BLUI1 & 32.10 & 3,23 & 1.75 \\
\hline Constant & & & -49.04 \\
\hline \multicolumn{4}{|l|}{ F2: canines } \\
\hline MDUC & 70.93 & 1,64 & 1.88 \\
\hline BLUC & 40.17 & 1,63 & 0.83 \\
\hline Constant & & & -16.93 \\
\hline \multicolumn{4}{|l|}{ F3: premolars } \\
\hline MDUP3 & 26.92 & 1,50 & 3.13 \\
\hline Constant & & & -14.25 \\
\hline \multicolumn{4}{|l|}{ F4: molars ${ }^{\mathrm{b}}$} \\
\hline MDUM2 & 16.47 & 1,35 & 1.69 \\
\hline Constant & & & -12.67 \\
\hline \multicolumn{4}{|c|}{ F5: molars (excluding M3) ${ }^{\mathrm{b}}$} \\
\hline MDUM2 & 27.21 & 1,50 & 1.25 \\
\hline BLUM1 & 20.49 & 2,49 & 1.17 \\
\hline Constant & & & -21.34 \\
\hline \multicolumn{4}{|c|}{ F6: incisors + canines } \\
\hline MDLI2 & 28.49 & 2,19 & 3.98 \\
\hline BLLC & 28.12 & 3,18 & 2.14 \\
\hline BLUI1 & 36.08 & 3,18 & 2.05 \\
\hline Constant & & & -44.29 \\
\hline \multicolumn{4}{|c|}{ F7: premolars + canines } \\
\hline MDUC & 30.54 & 1,36 & 1.59 \\
\hline MDUP3 & 18.52 & 2,35 & 1.73 \\
\hline Constant & & & -16.94 \\
\hline \multicolumn{4}{|c|}{ F8: molars + canines ${ }^{\mathrm{b}}$} \\
\hline MDUC & 19.19 & 1,26 & 2.64 \\
\hline Constant & & & -15.16 \\
\hline \multicolumn{4}{|c|}{ F9: molars + canines $\left(\right.$ excluding M3) ${ }^{\mathrm{b}}$} \\
\hline MDUC & 37.99 & 1,37 & 2.83 \\
\hline Constant & & & -16.21 \\
\hline
\end{tabular}

Method: Wilk's lambda with F: 3.84 to enter and F: 2.71 to remove

$M D$ mesiodistal, $B L$ buccolingual, $U$ upper, $L$ lower, $I$ incisor, $C$ canine, $P$ premolar, $M$ molar

${ }^{\mathrm{a}}$ The sectioning point for all the function is zero

${ }^{\mathrm{b}} \mathrm{M} 3$ was excluded from the discriminant function analysis

archaeological populations. It should be emphasised again that the present study has estimated the sex of the individuals using osteological methods.

All variables analysed here presented statistically significant differences between males and females $(p<0.001)$ with the exception of the maxillary third molars that were excluded
Table 5 Discriminant function analysis of cervical mesiodistal and buccolingual measurements for each tooth separately

\begin{tabular}{|c|c|c|c|}
\hline Variables entered $^{\mathrm{a}}$ & Exact $F$ & $\mathrm{df}$ & Raw coefficient \\
\hline \multicolumn{4}{|l|}{ F10: UI1 } \\
\hline MD & 12.25 & 1,50 & 0.82 \\
\hline $\mathrm{BL}$ & 27.14 & 1,50 & 2.33 \\
\hline Constant & & & -19.74 \\
\hline \multicolumn{4}{|l|}{ F11: LI1 } \\
\hline MD & 41.93 & 1,73 & 3.85 \\
\hline $\mathrm{BL}$ & 26.17 & 1,73 & 1.13 \\
\hline Constant & & & -19.79 \\
\hline \multicolumn{4}{|l|}{ F12: UI2 } \\
\hline MD & 14.91 & 1,53 & 0.95 \\
\hline $\mathrm{BL}$ & 23.66 & 1,53 & 1.99 \\
\hline Constant & & & -15.84 \\
\hline \multicolumn{4}{|l|}{ F13: LI2 } \\
\hline MD & 44.55 & 1,89 & 2.89 \\
\hline $\mathrm{BL}$ & 25.43 & 1,89 & 1.53 \\
\hline Constant & & & -20.36 \\
\hline \multicolumn{4}{|l|}{ F14: UC } \\
\hline MD & 78.52 & 1,82 & 1.87 \\
\hline $\mathrm{BL}$ & 61.91 & 1,82 & 0.92 \\
\hline Constant & & & -17.58 \\
\hline \multicolumn{4}{|l|}{ F15: LC } \\
\hline MD & 76.07 & 1,100 & 1.70 \\
\hline $\mathrm{BL}$ & 61.62 & 1,100 & 0.79 \\
\hline Constant & & & -14.91 \\
\hline \multicolumn{4}{|l|}{ F16: UP3 } \\
\hline MD & 44.87 & 1,85 & 3.05 \\
\hline $\mathrm{BL}$ & 13.45 & 1,85 & -0.04 \\
\hline Constant & & & -13.57 \\
\hline \multicolumn{4}{|l|}{ F17: LP3 } \\
\hline MD & 67.10 & 1,109 & 2.75 \\
\hline $\mathrm{BL}$ & 42.87 & 1,109 & 0.54 \\
\hline Constant & & & -16.71 \\
\hline \multicolumn{4}{|l|}{ F18: UP4 } \\
\hline MD & 35.07 & 1,81 & 2.25 \\
\hline $\mathrm{BL}$ & 24.81 & 1,81 & 0.53 \\
\hline Constant & & & -14.88 \\
\hline \multicolumn{4}{|l|}{ F19: LP4 } \\
\hline MD & 43.24 & 1,102 & 2.35 \\
\hline $\mathrm{BL}$ & 22.80 & 1,102 & 0.53 \\
\hline Constant & & & -15.55 \\
\hline \multicolumn{4}{|l|}{ F20: UM1 } \\
\hline MD & 35.14 & 1,82 & 1.43 \\
\hline $\mathrm{BL}$ & 37.80 & 1,82 & 1.16 \\
\hline Constant & & & -22.61 \\
\hline \multicolumn{4}{|l|}{ F21: LM1 } \\
\hline MD & 31.62 & 1,94 & 0.96 \\
\hline $\mathrm{BL}$ & 39.11 & 1,94 & 1.25 \\
\hline Constant & & & -19.51 \\
\hline
\end{tabular}

$M D$ mesiodistal, $B L$ buccolingual, $U$ upper, $L$ lower, $I$ incisor, $C$ canine, $P$ premolar, $M$ molar

${ }^{\mathrm{a}}$ The sectioning point for all the functions is zero

from the analysis. A comparison between the two sexes showed that the classification accuracy of all functions was higher for males. This result is in agreement with other studies on cervical tooth measurements (Vodanovic et al. 2007; Hassett 2011; Viciano et al. 2011, 2013, 2015; Zorba et al. 2011, 2013; Mujib et al. 2014; Peckmann et al. 2015). This means that females have a greater variation in tooth size and can more often be misclassified as male. 
Table 6 Discriminant function analysis of cervical mesiodistal and buccolingual measurements for each tooth separately

\begin{tabular}{llll}
\hline Variables entered $^{\mathrm{a}}$ & Exact $F$ & $\mathrm{df}$ & Raw coefficient \\
\hline F22: UM2 & & & \\
MD & 56.44 & 1,80 & 0.96 \\
BL & 65.33 & 1,80 & 1.07 \\
Constant & & & -17.83 \\
F23: LM2 & & & \\
MD & 54.47 & 1,105 & 1.00 \\
BL & 62.15 & 1,105 & 1.13 \\
Constant & & & -18.34 \\
F24: LM3 & & & \\
MD & 9.98 & 1,67 & 0.51 \\
BL & 24.08 & 1,67 & 1.63 \\
Constant & & & -17.49 \\
\hline
\end{tabular}

$M D$ mesiodistal, $B L$ buccolingual, $U$ upper, $L$ lower, $I$ incisor, $C$ canine, $P$ premolar, $M$ molar

${ }^{\mathrm{a}}$ The sectioning point for all the function is zero

${ }^{\mathrm{b}} \mathrm{M} 3$ was excluded from the discriminant function analysis
The greatest difference in percentage of sexual dimorphism was observed in canine mesiodistal measurements. There is little comparative data against which the amount of sexual dimorphism in the cervical measurements can be compared. Only Vodanovic et al. (2007) and Tuttösí and Cardoso (2015) provided percentage of sexual dimorphism for cervical tooth measurements in other archaeological samples. Vodanovic et al. (2007), however, reported only the SD\% for mesiodistal measurements of maxillary canine and mandibular third molar. The SD\% for maxillary canine mesiodistal measurement was $13.93 \%$, which is similar to the Tuttösí and Cardoso (2015) study (13.83\%) and about $4 \%$ higher compared to the Vodanovic et al. (2007) study $(9.55 \%)$. The highest percentage of sexual dimorphism was observed in mandibular canine. The SD\% for this tooth was $14.99 \%$ (Mesiodistal) and $12.35 \%$ (Buccolingual), which are significantly higher compared to the Tuttösí and Cardoso (2015) study (4.90\% mesiodistal and $6.87 \%$ buccolingual). In the latter study, maxillary second incisor showed the highest percentage of sexual
Table 7 Classification accuracy of original and cross-validated samples

\begin{tabular}{|c|c|c|c|c|c|c|c|}
\hline \multirow[t]{3}{*}{ Functions } & \multicolumn{6}{|c|}{ Predicted group membership } & \multirow[t]{3}{*}{$N$} \\
\hline & \multicolumn{3}{|c|}{ Original (\%) } & \multicolumn{3}{|c|}{ Cross-validated (\%) } & \\
\hline & Male & Female & Total & Male & Female & Total & \\
\hline F1: incisors & 100 & 100 & 100 & 100 & 100 & 100 & 27 \\
\hline F2: canines & 92.5 & 76.9 & 86.4 & 90 & 76.9 & 84.8 & 66 \\
\hline F3: premolars & 79.3 & 69.6 & 75 & 79.3 & 69.6 & 75 & 52 \\
\hline F4: molars & 88.5 & 45.5 & 75.7 & 88.5 & 45.5 & 75.7 & 37 \\
\hline F5: molars (excluding M3) & 85.7 & 76.5 & 82.7 & 85.7 & 76.5 & 82.7 & 52 \\
\hline F6: incisors + canines & 100 & 100 & 100 & 100 & 100 & 100 & 22 \\
\hline F7: premolars + canines & 87.5 & 78.6 & 84.2 & 87.5 & 78.6 & 84.2 & 38 \\
\hline F8: molars + canines & 95.2 & 71.4 & 89.3 & 95.2 & 71.4 & 89.3 & 28 \\
\hline F9: molars + canines (excluding M3) & 96.4 & 81.8 & 92.3 & 96.4 & 81.8 & 92.3 & 39 \\
\hline F10: UI1 & 80 & 72.7 & 76.9 & 80 & 72.7 & 76.9 & 52 \\
\hline F11: LI1 & 89.6 & 77.8 & 85.3 & 87.5 & 77.8 & 84 & 75 \\
\hline F12: UI2 & 94.1 & 66.7 & 83.6 & 91.2 & 66.7 & 81.8 & 55 \\
\hline F13: LI2 & 91.5 & 81.3 & 87.9 & 91.5 & 78.1 & 86.8 & 91 \\
\hline F14: UC & 90 & 82.4 & 86.9 & 88 & 82.4 & 85.7 & 84 \\
\hline F15: LC & 90.9 & 77.8 & 86.3 & 90.9 & 77.8 & 86.3 & 102 \\
\hline F16: UP3 & 82.7 & 65.7 & 75.9 & 82.7 & 62.9 & 74.7 & 87 \\
\hline F17: LP3 & 85.5 & 69 & 79.3 & 84.1 & 69 & 78.4 & 111 \\
\hline F18: UP4 & 81.6 & 70.6 & 77.1 & 81.6 & 67.6 & 75.9 & 83 \\
\hline F19: LP4 & 88.2 & 61.1 & 78.8 & 86.8 & 61.1 & 77.9 & 104 \\
\hline F20: UM1 & 88.2 & 72.7 & 82.1 & 86.3 & 69.7 & 79.8 & 84 \\
\hline F21: LM1 & 87.1 & 61.8 & 78.1 & 87.1 & 61.8 & 78.1 & 96 \\
\hline F22: UM2 & 86.5 & 76.7 & 82.9 & 86.5 & 73.3 & 81.7 & 82 \\
\hline F23: LM2 & 87 & 76.3 & 83.2 & 87 & 73.7 & 82.2 & 107 \\
\hline F24: LM3 & 88.9 & 54.2 & 76.8 & 84.4 & 54.2 & 73.9 & 69 \\
\hline
\end{tabular}

$M D$ mesiodistal, $B L$ buccolingual, $U$ upper, $L$ lower, $I$ incisor, $C$ canine, $P$ premolar, $M$ molar 


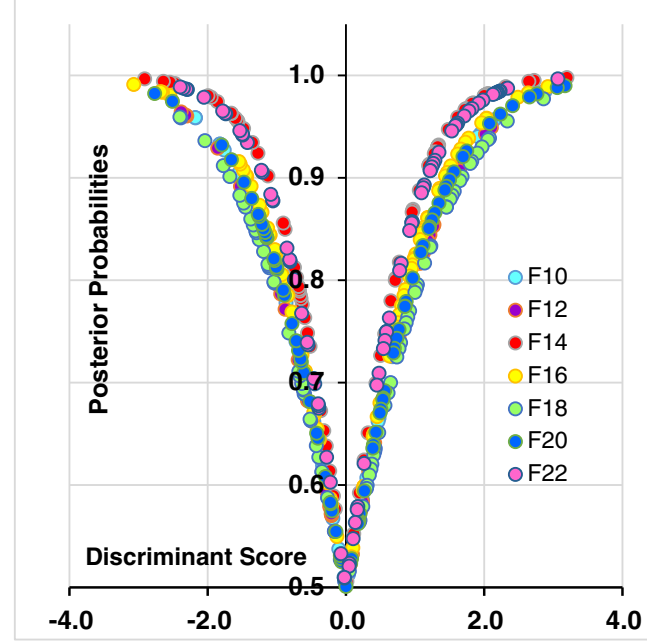

Fig. 1 Probability levels of correct sexing for each individual, single tooth analysis (maxillary teeth)

dimorphism contradicting the present and other studies (Cardoso 2008; Zorba et al. 2011). For the molar teeth, second molar showed the highest percentage of sexual dimorphism in accordance with the results of Tuttösí and Cardoso (2015) and also those of crown measurement studies (Cardoso 2008; Garn et al. 1979; Zorba et al. 2011).

Discriminant function analysis for single tooth measurements also showed that the cervical measurements of the permanent canines and incisors were the most dimorphic teeth providing classification accuracy between 76.9 and $87.9 \%$. These results were in accordance with previous studies (Alt et al. 1998; Starp 1990; Ellendt 1993; Hassett 2011; Viciano et al. 2011, 2013, 2015; Mujib et al. 2014). In addition, it was found that second molar dimensions can be a very effective single variable for sex estimation with a classification accuracy of $83 \%$. A similar result was achieved for a Modern Greek population (Zorba et al. 2012), and some archaeological populations (Starp 1990; Ellendt 1993; Viciano et al. 2015;

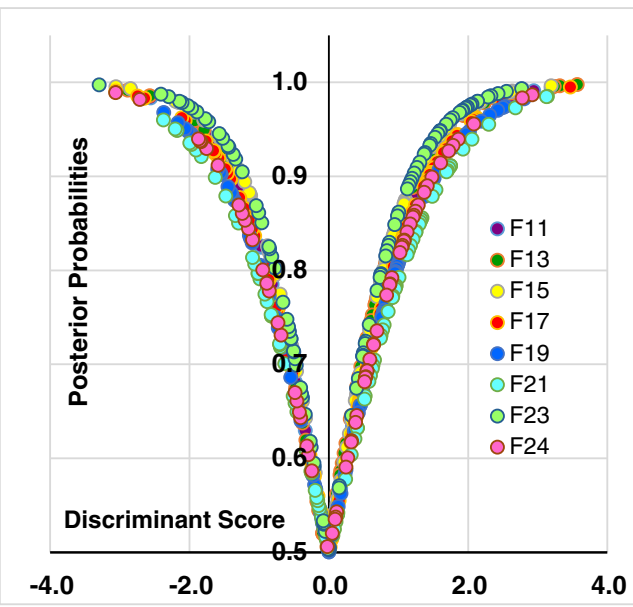

Fig. 2 Probability levels of correct sexing for each individual, single tooth analysis (mandibular teeth)

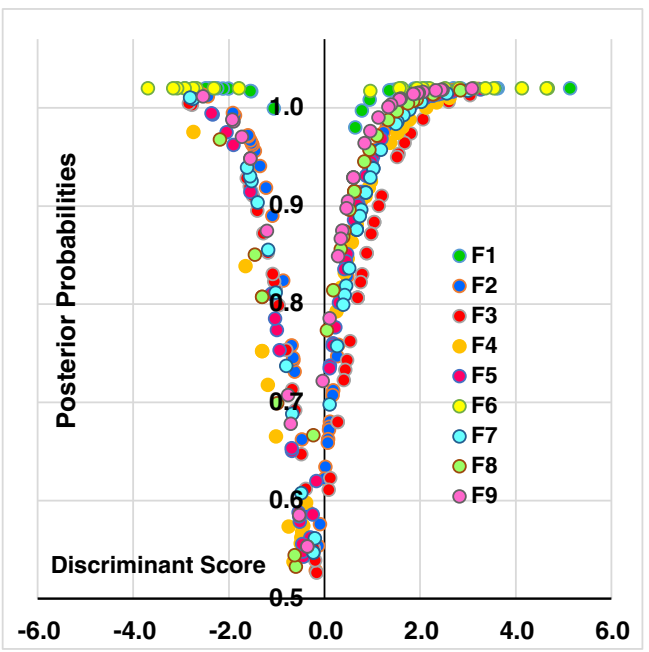

Fig. 3 Probability levels of correct sexing for each individual, functions $1-9$

Tuttösí and Cardoso 2015) also reported a high percentage of correct classification for second molar.

Furthermore, several different discriminant functions were created using different combinations of tooth dimensions. The best discriminant functions for sex classification used maxillary and mandibular incisors (F1) and a combination of maxillary and mandibular incisors and canines (F6). The obtained classification accuracy rates were $100 \%$ for both original and cross-validated data; however, this observation was based on a small sample size $(n=27, n=22)$, and despite the fact that functions derived from similar size samples are reported (e.g. Viciano et al. 2011), we do not recommend the use of this equation for sex estimation without a follow-up study on a larger sample. The second best discriminant function used a combination of canines, first molars and second molars (F9) with an accuracy rate of $92.3 \%$ for both original and crossvalidated data. This was followed by a discriminant function analysis that used maxillary and mandibular canines providing a correct sex classification of $86.4 \%$ for the original and $84.8 \%$ for the cross-validated data. Although Ișcan and Kedici (2003) reported that the majority of difference between the sexes appears to come from the canines, Garn et al. (1967) suggested that the teeth located adjacent to the canines are more dimorphic than others; however, some studies of crown mesiodistal and buccolingual measurements indicated that incisors are the least sexually dimorphic teeth (Bishara et al. 1989, Ling and Wong 2007). Acharya and Mainali (2007), however, found that central and lateral incisors show significant sexual dimorphism.

In conclusion, sex estimation using dental cervical measurements in an Iranian population has proven to be highly accurate for both original and cross-validated data. It must be stressed though, that the expression of sexual dimorphism was calculated based on the individuals for which sex could be accurately estimated. This means that the sample may not be 
totally representative of the population, which introduces a bias in the analysis. Nevertheless, this study is of importance for the application to unknown skeletal remains from Iran around the same period (Iron Age); especially considering that they are more likely to survive harsh taphonomic conditions than any other skeletal element (Andersen et al. 1995; Vodanovic et al. 2007; Fereira et al. 2008). It is highly recommended to consider reliable estimates with over $95 \%$ probability of correct classification. For estimates with 80-95\% probability, the prediction should be treated with caution, while any estimate with probability lower than $80 \%$ should be considered unreliable and alternative methods should be used. Moreover, the percentage of accuracy may be slightly inflated due to small sample sizes used in some of the functions. This is why the authors aim to do additional testing on an expanded data set as a direction for future research.

Acknowledgments We would like to thank Dr. Janet Monge for providing us access to the Hasanlu and Dinkha Tepe skeletal collections at the University of Pennsylvania Museum of Archaeology and Anthropology. We would also like to thank Dr. Linda Fibiger for her valuable contribution and support during the examination process and Caroline Lille for her helpful suggestions.

Open Access This article is distributed under the terms of the Creative Commons Attribution 4.0 International License (http://creativecommons.org/ licenses/by/4.0/), which permits unrestricted use, distribution, and reproduction in any medium, provided you give appropriate credit to the original author(s) and the source, provide a link to the Creative Commons license, and indicate if changes were made.

\section{References}

Acharya AB, Mainali S (2007) Univariate sex dimorphism in Nepalese dentition and the use of discriminant function in gender assessment. Forensic Sci Int 173:47-56

Alt KW, Riemensperger B, Vach W, Krekeler G (1998) Zahnwurzellänge und Zahnhalsdurchmesser als Indikatoren zur Geschlechtsbestimmung an Menschlichen Zähnen. Anthrop Anz 56:131-144

Andersen L, Juhl M, Solheim T, Borrman H (1995) Odontological identification of fire victims - potentialities and limitations. Int J Legal Med 107:229-234

Ateș M, Karaman F, Ișcan MY, Erdem TL (2006) Sexual differences in Turkish dentition. Legal Med 8(5):288-292

Bishara SE, Jakobsen JR, Abdillah EM, Fernandez Garcia A (1989) Comparisons of mesiodistal and buccolingual crown dimensions of the permanent teeth in three populations from Egypt, Mexico, and the United States. Am J Orthod Dentofac Orthop 96(5):416-422

Black TK (1978) Sexual dimorphism in the tooth-crown diameters of the deciduous teeth. Am J Phys Anthropol 48:77-82

Brooks ST, Suchey JM (1990) Skeletal age determination based on the Os pubis: a comparison of the Acsddi-Nemeskeri and Suchey-brooks methods. Hum Evol 5:227-238

Buckberry JL, Chamberlain AT (2002) Age estimation from the auricular surface of the ilium: a revised method Am. J Phys Anthropol 119: 231-239

Buikstra JE, Ubelaker DH (1994) Standards for data collection from human skeletal remains. Arkansas Archeological Society, Fayettville
Cardoso HFV (2008) Sample-specific (universal) metric approached for determining the sex of immature human skeletal remains using permanent tooth dimensions. J Archaeol Sci 35:158-168

Ditch LE, Rose JC (1972) A multivariate dental sexing technique. Am J Phys Anthropol 37:61-64

Dyson RH (1965) Problems of Protohistoric Iran as seen from Hasanlu. JNES 24:193-217

Ellendt, G., 1993. Untersuchungen zur Geschlechtsbestimmung der Skelettreste zweier schnurkeramischer Gräberfelder der Jungsteinzeit aus Baden-Württemberg anhand der Zahnmaße. Dissertation, Universitat Tübingen.

Fereira JI, Fereira AE, Ortega AI (2008) Methods for the analysis of hard dental tissues exposed to high temperatures. Forensic Sci Int 178:119-124

Garn SM, Lewis AB, Kerewsky RS (1964) Sex differences in tooth size. J Dent Res 43(2):306

Garn SM, Kerewsky RS, Swindler DR (1966) Canine "field" in sexual dimorphism of tooth size. Nature 212:1501-1502

Garn SM, Lewis AB, Swindler DR, Kerewsky RS (1967) Genetic control of sexual dimorphism in tooth size. J Dent Res 46:963-972

Garn SM, Cole P, Van Alstine W (1979) Sex discriminatory effectiveness using combinations of root lengths and crown diameters. Am J Phys Anthropol 50:115-118

Gonçalves D, Granja R, Cardoso F, de Carvalho AF (2014) Samplespecific sex estimation in archaeological contexts with commingled human remains: a case study from the middle Neolithic cave of Bom Santo in Portugal. J Archaeol Sci 49:185-191

Hassett B (2011) Technical note: estimating sex using cervical canine odontometrics: a test using a known sex sample. Am J Phys Anthropol 146:486-489

Hattab FN, Al-Momani AS, Yassin OM (1997) Odontometric study of deciduous and permanent teeth in Jordanians. Dental Morphology 4:17-24

Hillson S, FitzGerald C, Flinn H (2005) Alternative dental measurements: proposals and relationships with other measurements. Am J Phys Anthropol 126:413-426

Hughes TE, Townsend GC (2013) Twin and family studies of human dental crown morphology: genetic, epigenetic, and environmental determinations of modern human dentitions. In: Scott GR, Irish JD (eds) Anthropological prospective on tooth morphology, genetics, evolution, variation. Cambridge University Press, Cambridge, pp. 31-68

Ișcan MY, Kedici PS (2003) Sexual variation in buccolingual dimensions in Turkish dentition. Forensic Sci Int 137(2-3):160-164

Khamis MF, Taylor JA, Malik SN, Townsend GC (2014) Odontometric sex variation in Malaysians with application to sex prediction. Forensic Sci Int 234:183 e1-7

Kieser JA (1990) Human adult odontometrics. Cambridge University Press, Cambridge

Kieser JA, Geroenveld HT, Preston CB (1985) A metrical analysis of the south African Caucasoid dentition. J Dent Assoc S Afr 40:121-125

Kondo S, Townsend GC (2004) Sexual dimorphism in crown units of mandibular deciduous and permanent molars in Australian Aborigines. Homo 55(1-2):53-64

Kranioti EF, Ișcan MY, Michalodimitrakis M (2008) Craniometric analysis of the modern Cretan population. Forensic Sci Int 180(2-3):110. e1-5

Ling JYK, Wong RWK (2007) Tooth dimensions of southern Chinese. Homo 58:67-73

Meindl RS, Lovejoy CO (1985) Ectocranial suture closure: a revised method for the determination of skeletal age at death based on the lateral-anterior sutures. Am J Phys Anthropol 68:57-66

Miles AEW (2001) The miles method of assessing age from tooth wear revisited. J Archaeol Sci 28:973-982

Mitsea AG, Moraitis K, Leon G, Nicopulou-Karayianni K, Spiliopoulou C (2014) Sex determination by tooth size in a sample of Greek population. Homo 65(4):322-329

Mujib ABR, Tarigoppula RVN, Kulkarni PG, Anil BS (2014) Gender determination using diagonal measurements of maxillary molar and canine teeth in Davangere population. J Clin Diagn Res 8(11):ZC141-ZC144 
Muscarella OW (1974) The Iron Age at Dinkha Tepe, Iran. Metrop Mus J 9:35-90

Muscarella OW (1988) Bronze and iron: Ancient near Eastern artifacts in The metropolitan museum of art. Metropolitan Museum of Art, New York

Peckmann TR, Meek S, Dilkie N, Mussett M (2015) Sex estimation using diagonal diameter measurements of molar teeth in African American populations. J Forensic Legal Med 36:70-80

Pereira C, Bernardo M, Pestana D, Santos J, Mendonca MC (2010) Contribution of teeth in human forensic identification- discriminant function sexing odontometrical techniques in Portuguese population. J Forensic Legal Med 17(2):105-110

Phenice TW (1969) A newly developed visual method of sexing in the os pubis. Am J Phys Anthropol 30:297-301

Prabhu S, Acharya AB (2009) Odontometric sex assessment in Indians. Forensic Sci Int 192:129e1-129e5

Sharma S, Dinkar AD, Bedi S (2015) Odontometric sexual dimorphism: a sibling correlation. J Clin Diagn Res 8(3):233-235

Starp, S.E., (1990). Untersuchungen zur Geschlechtsbestimmung der Individuen zweier frühneolithischer Skelettserien aus BadenWürttemberg anhand der Zahnmaße. Dissertation, Universitat Tübingen.

Townsend G, Brook A, Yong R, Hughes T (2016) Tooth classes, field concepts, and symmetry. In: Irish JD, Scott GR (eds) A companion to dental anthropology. John Wiley \& Sons, West Sussex, pp. 172 189

Tuttösí P, Cardoso HFV (2015) An assessment of sexual dimorphism and sex estimation using cervical dental measurements in a northwest coast archaeological sample. J Archaeol Sci: Report 3:306-312
Ulijaszek SJ, Kerr AD (1999) Anthropometric measurement error and the assessment of nutritional status. Br J Nutr 82(3):165-177

Viciano J, Aleman I, D’Anastasio R, Capasso L, Botella MC (2011) Odontometric sex discrimination in the Herculaneum sample (79 AD, Naples, Italy), with application to juveniles. Am J Phys Anthropol 145:97-106

Viciano J, Lopez-Lazaro S, Aleman I (2013) Sex estimation based on deciduous and permanent dentition in a contemporary Spanish population. Am J Phys Anthropol 152(1):31-43

Viciano J, D'Anastasio R, Capasso L (2015) Odontometric sex estimation on three populations of the iron age from Abruzzo region (centralsouthern Italy). Arch Oral Biol 60(1):100-115

Vodanovic M, Demo Z, Njemirovskij V, Keros J, Brkic H (2007) Odontometrics: a useful method for sex determination in an archaeological skeletal population? J Archaeol Sci 34:905913

Walker PL (2008) Sexing skulls using discriminant function analysis of visually assessed traits. Am J Phys Anthropol 136:39-50

Yuen KK, So LL, Tang EL (1997) Mesiodistal crown diameters of the primary and permanent teeth in southern Chinese - a longitudinal study. Eur J Orthod 19(6):721-731

Zorba E, Moraitis K, Manolis SK (2011) Sexual dimorphism in permanent teeth of modern Greeks. Forensic Sci Int 210:74-81

Zorba E, Moraitis K, Eliopoulos C, Spiliopoulou C (2012) Sex determination in modern Greeks using diagonal measurements of molar teeth. Forensic Sci Int 217:19-26

Zorba E, Spiliopoulou C, Moraitis K (2013) Evaluation of the accuracy of different molar teeth measurements in assessing sex. Forensic Sci Med Pathol 9:13-23 